\title{
Bioenergie-Regionen stärken
}

\author{
Die Produktion und Bereitstellung holzbasierter \\ Biomasse zur Energiegewinnung ist ein Baustein \\ der Energiewende. Dabei spielen so unterschied- \\ liche Herausforderungen wie Flächenverfüg- \\ barkeit, ökonomische Konkurrenzfähigkeit gegen- \\ über anderen Landnutzungsformen bis hin \\ zur ökologischen Verträglichkeit von Energieholz \\ eine Rolle. \\ Von Michael Bredemeier, Norbert P. Lamersdorf \\ und Christian Ammer
}

\section{Hintergrund und Aufgabenstellung}

Die Vorräte fossiler Brennstoffe sind bekanntermaßen endlich, daher müssen in der Energieversorgung neue und vielfältige Wege gefunden und gegangen werden. Die Energiewende soll es ermöglichen, Energie nachhaltig, zu vertretbaren Kosten und möglichst ohne große Transportverluste am Ort des Verbrauchs bereitzustellen. Dabei soll die Produktion neuer, alternativer Energien umweltverträglich erfolgen, also Ökosysteme und die von ihnen ausgehenden Leistungen nicht beeinträchtigen. Diese Vision wurde unter anderem im Nationalen Biomasseaktionsplan für Deutschland formuliert.

Biomasse ist aktuell der bedeutendste erneuerbare Energieträger in Deutschland. Im Jahr 2013 betrug der Anteil der Bioenergie an der gesamten Bereitstellung von erneuerbaren Energien 62\%. Neben der Bioenergie zählen unter anderem Wind-, Solar- und geothermische Energie zu den sogenannten Erneuerbaren. Zur Energiegewinnung aus Biomasse werden hauptsächlich Holz, landwirtschaftliche Energiepflanzen wie Mais, Getreide und Raps, landwirtschaftliche Reststoffe sowie Bioabfälle genutzt. Insgesamt lässt sich feststellen, dass die Gewinnung von Energie aus Biomasse einen wichtigen Beitrag zur angestrebten nachhaltigen und klimaneutralen Energieversorgung leisten kann, wenngleich nicht alle Produktionssysteme gleichermaßen effizient und im Hinblick auf Folgewirkungen unbedenklich sind.

Im Rahmen des Forschungsvorhabens BEST wurden die wesentlichen im Nationalen Biomasseaktionsplan für Deutschland enthaltenen Ziele aufgegriffen. Dazu zählen insbesondere die Entwicklung integrativer Konzepte zur nachhaltigen Landnutzung, die Berücksichtigung kulturlandschaftlicher Wirkun- gen, die Vermeidung von Umweltbelastungen, die Erschließung von ungenutzten Potenzialen zur Energiebereitstellung und Vorschläge zur effizienten Lösung divergierender Nutzungsinteressen.

Vor diesem Hintergrund hatte sich das Verbundprojekt die Aufgabe gestellt, die sich aus dem Biomasseaktionsplan für Deutschland ergebenden Erfordernisse zu konkretisieren und auf regionaler Ebene für zwei Beispielregionen umfassende wissenschaftliche und praktische Beiträge zu folgenden Aspekten zu leisten:

- divergierende Landnutzungsinteressen durch eine Optimierung der bestehenden Konzepte und Wertschöpfungsketten auszugleichen und/oder durch innovative neue Konzepte aufzulösen,

- eine ökologische und sozio-ökonomische Bewertung möglicher Handlungsalternativen vorzunehmen,

- gemeinsam mit Nutzer/innen und Betroffenen („Stakeholdern“) konkrete, regionale und rationale Handlungsoptionen zu entwickeln und sie zielgruppengerecht an die Landnutzer/innen zu kommunizieren beziehungsweise durch Demonstrationsobjekte zu etablieren.

Regionale Landnutzungsentscheidungen müssen im Spannungsfeld von Ernährung, Energie- und Rohstoffversorgung, Arbeitsplatzsicherung und der Notwendigkeit zur Bereitstellung ökosystemarer Dienstleistungen getroffen werden. Zusätzlich ist die Landnutzung damit konfrontiert, sich an den Klimawandel anpassen zu müssen. Dies erschwert die Entscheidung für oder gegen eine bestimmte Art der Landnutzung erheblich, da im Gegensatz zu früher nicht mehr von weitgehend konstanten Umweltbedingungen ausgegangen werden kann. Von den damit zusammenhängenden Schwierigkeiten sind die in jüngster Zeit in Deutschland gebildeten Bioenergie-Regionen besonders betroffen. Sie haben zwar einerseits angesichts der Möglichkeit, durch nachwachsende Rohstoffe fossile Energieträger zu substituieren für die künftige Entwicklung anderer Regionen eine beispielgebende Bedeutung. Andererseits verschärft die Notwendigkeit, Biomasse für die energetische Nutzung zu produzieren, die Konkurrenz zwischen den verschiedenen Optionen der Landnutzung.

Die Produktion von Biomasse für die energetische Nutzung ist eine klimapolitisch besonders attraktive Form der Landnutzung. Sie ist dadurch gekennzeichnet, dass der ländliche Raum Flächen und Bewirtschaftungsinstrumente für die Biomassenproduktion bereitstellt, während die daraus erzeugte Energie insbesondere im urbanen Raum verbraucht wird. 
Biomasse kann in naher Zukunft wesentlich zur Sicherung der Energieversorgung beitragen. Entsprechend hoch ist seit Beginn des 21. Jahrhunderts die Dynamik des landwirtschaftlichen Biomasseausbaus, insbesondere in den Bereichen Kraftstoff (Biodiesel) und Stromerzeugung (Biogas). Stark zunehmend zeigt sich auch die im Wesentlichen über holzige Biomasse aus dem Wald gespeiste Entwicklung auf dem Wärmemarkt. Direkte Anreize zum verstärkten Einsatz von Biomasse als nachwachsendem Rohstoff liefern unter anderen das neu ausgerichtete Erneuerbare-Energien-Gesetz (EEG), das Erneuerbare-Energien-Wärmegesetz (EEWärmeG), Steuervergünstigungen, Investitionsförderungen sowie indirekt die zumindest mittelfristig wieder zu erwartende Verknappung und Verteuerung von Mineralöl. Mit Blick auf mögliche Weiterentwicklungen im Bereich der Biokraftstoffe der zweiten Generation und einer entsprechend steigenden Nachfrage nach holziger Biomasse geraten zunehmend auch Gehölzbestände, entweder als Wald im klassischen Sinne oder als Kurzumtriebsplantagen (KUP) mit schnellwachsenden Baumarten auf landwirtschaftlicher Fläche als potenzielle Rohstofflieferanten in den Blickpunkt der Betrachtung.

Sowohl im Landwirtschafts- als auch im Forstsektor zeigen sich jedoch auch erste Konflikte zwischen den verschiedenen Interessenvertretern (Produzent/innen, Abnehmer/innen, Planungs- und Genehmigungsbehörden, Natur- und Umweltschutzorganisationen, Politik) bezüglich zukünftiger Nutzungskonzeptionen. Ein Beispiel hierfür ist die nach wie vor ungeklärte Frage, welchen Anteil die Bioenergieerzeugung aus Holz zukünftig einnehmen soll. So konkurriert eine aus kohlenstoffökologischer Sicht vorteilhafte Steigerung der Holznutzung zum Zwecke der Energieerzeugung wegen der damit verbundenen Substitutionseffekte unter Umständen mit der Nahrungsmittelproduktion bei Nutzung landwirtschaftlicher Flächen, der Erholungsnutzung oder dem Arten- und Biotopschutz bei der Waldnutzung. In dieser Diskussion wird zunehmend deutlicher, dass die primär sektoral geprägten Vorstellungen und Regelungen der Landnutzung nicht mehr ausreichen, um den Herausforderungen einer möglichst effektiven, ressourcenschonenden, regional-adaptiven und nachhaltigen Erzeugung von Biomasse gerecht zu werden. Gefordert ist vielmehr eine vergleichende Bewertung unterschiedlicher Wertschöpfungsketten in ökologischer, ökonomischer und sozialer Hinsicht. Konkret ist es hierzu erstens nötig, potenziell vorhandene Biomassereserven zu identifizieren, zweitens denkbare Anbausysteme hinsichtlich der genannten Aspekte zu bewerten und drittens Möglich- keiten einer Kaskadennutzung zu untersuchen. An genau diesen Punkten setzte das Verbundprojekt BEST an.

\section{Planung und Ablauf}

BEST als Verbundprojekt der Georg-August-Universität Göttingen und neun weiterer Institutionen und Einrichtungen umfasste insgesamt 31 Teilprojekte, die den folgenden sieben übergreifenden thematischen Clustern zugeordnet waren:

- ökologische Landschaftsfunktionen,

【 Systemlösungen für die Mobilisierung von Holzreserven,

- innovative Anbausysteme und -verfahren,

- Systemlösungen für eine stofflich-energetische Kaskadennutzung,

I ökologische Folgenabschätzung unterschiedlicher Bewirtschaftungsformen,

I sozioökonomische Bewertung von Nutzungskonzepten,

- Ergebnisintegration, Umsetzung und Partizipation.

Dem integrativen Ansatz folgend wurden unterschiedliche Nutzungskonzepte formuliert, umfassend bewertet und mit Stakeholdern vielfach diskutiert. Die Ergebnisse aus den entsprechenden Teilprojekten wurden seitens der Projektkoordination integriert, im Hinblick auf die jeweiligen Adressaten verdichtet und unter anderem über sogenannte Regionalkonferenzen an die beteiligten Akteure kommuniziert. Zum Ende der Projektlaufzeit konnte eine gemeinsame Buchveröffentlichung mit einem verwandten Partnerprojekt realisiert werden [1].

\section{Ergebnisse}

\subsection{Grundwasserneubildung (GWN)}

Kurzumtriebsplantagen (KUP) zur Energieholzproduktion haben aufgrund ihrer hohen Produktivität einen hohen Wasserverbrauch. Es galt deshalb räumlich explizit zu prüfen, wo dieser gedeckt werden kann beziehungsweise ob Abfluss und Grundwasserneubildung dort, wo sie erwünscht sind, durch den Anbau von KUP nicht gefährdet werden. Tabelle 1 zeigt die modellierten Grundwasserneubildungsmengen (GWN) unter Acker und unter Pappel-KUP mit und ohne Rotation, jeweils als Mittel über die Periode 1981-2010, sowie modelliert über die fünf trockensten und fünf feuchtesten Jahre dieser Periode. Es zeigt sich, dass ohne Berücksichtigung der KUP-spezifischen Rotation (simuliert als jährlich konstante dreijährige KUP) die GWN deutlich unterschätzt beziehungsweise die Evapotran-

\begin{tabular}{|c|c|c|c|c|c|c|c|}
\hline & & mittel (19 & 2010) & trocken $(5$ & ire) & feucht (5) & \\
\hline Niederschlag [mm] & & $693( \pm 118$ & & $551( \pm 44)$ & & $881( \pm 91)$ & \\
\hline Grundwasserneubildung (GWN) [mm] & Acker & $150( \pm 68)$ & $100 \%$ & $65( \pm 27)$ & $100 \%$ & $230( \pm 67)$ & $100 \%$ \\
\hline & KUP ohne Rotation & $119( \pm 77)$ & $79 \%$ & $28( \pm 20)$ & $43 \%$ & $182( \pm 74)$ & $79 \%$ \\
\hline & KUP mit Rotation & $139( \pm 79)$ & $93 \%$ & $47( \pm 35)$ & $72 \%$ & $213( \pm 73)$ & $93 \%$ \\
\hline
\end{tabular}

Tabelle 1: Grundwasserneubildung von Pappel-KUP ohne Rotation und in 5-jähriger Rotation im Vergleich zu Acker als Mittel über die Periode 1981-2010, sowie als Mittel über die fünf trockensten und fünf feuchtesten Jahre dieser Periode. 


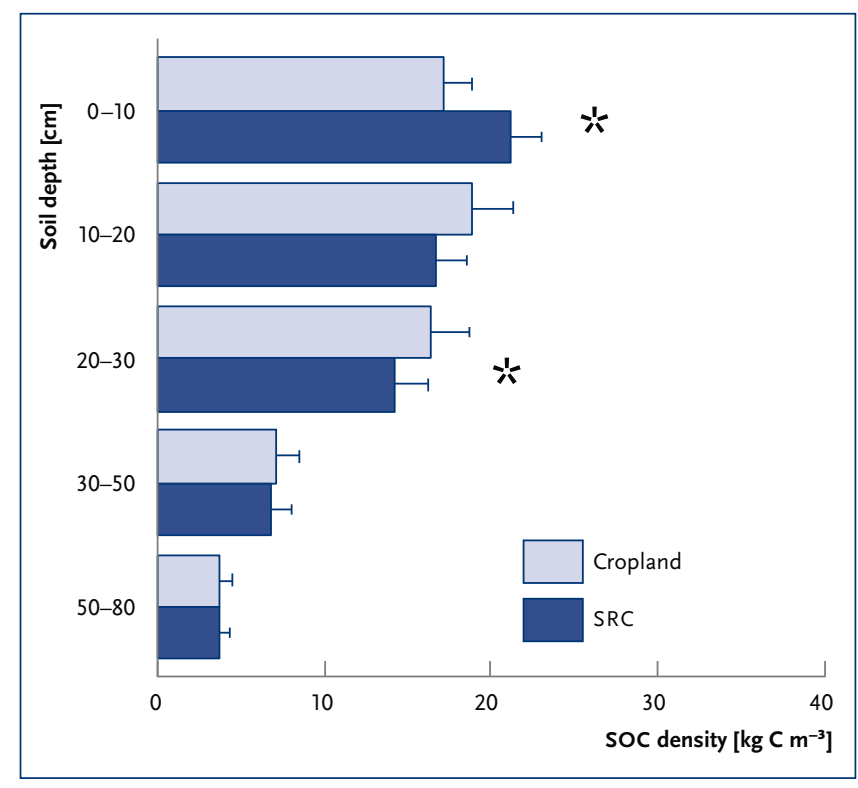

Abbildung 1: Organischer Bodenkohlenstoff von Acker und KUP in den fünf untersuchten Tiefenstufen

(Quelle: Walter, Don und Flessa 2015, Teilprojekt ÖL 3 im BEST-Abschlussbericht)

spiration überschätzt wird. Realistischer, das heißt unter Berücksichtigung einer fünfjährigen Rotation, verringert sich die GWN unter KUP im Vergleich zu Acker im Mittel (19812010) sowie in feuchten Jahren um nur $7 \%$. In trockenen Jahren ist die Abnahme mit $28 \%$ deutlicher. Dabei kann es in trockenen Jahren auch zum vollständigen Ausbleiben der GWN unter KUP kommen. In feuchten Jahren ist die GWN unter KUP im Vergleich zur sonstigen landwirtschaftlichen Nutzung ebenfalls geringer, bleibt aber nie aus. Daraus lässt sich folgern, dass in Regionen mit einer bereits unter den gegenwärtigen Klimabedingungen angespannten Wasserhaushaltssituation der Anbau von KUP kritisch geprüft werden sollte, während er in gut wasserversorgten Gegenden unkritisch ist. Tabelle 1 lässt auch Regulierungsmöglichkeiten durch die KUP-spezifische Rotationsbewirtschaftung vermuten, zum Beispiel durch Änderungen in der Länge der Rotationszeiten, angepasst an zurückliegende Trocken- und Feuchtjahre.

\subsection{Treibhausgasemissionen}

Bei der Untersuchung von Treibhausgasemissionen und Kohlenstoffbilanzen verschiedener Landnutzugstypen zeigte sich, dass die $\mathrm{N}_{2} \mathrm{O}$-Emissionen der KUPs meist deutlich unter jenen der agrarischen Landnutzungssysteme lagen.

Bei der Etablierung von KUP auf Acker verändert sich die Verteilung des organischen Bodenkohlenstoffs (OBK): während es in den obersten $10 \mathrm{~cm}$ zu einer signifikanten Akkumulation von OBK kommt, nimmt der OBK in $20-30 \mathrm{~cm}$ signifikant ab (siehe Abbildung 1). Diese Umverteilung ist vor allem auf die ausbleibende Bodenbearbeitung unter KUP zurückzuführen. Die gesamten OBK-Vorräte in $0-80 \mathrm{~cm}$ unter KUP verändern sich hingegen nicht signifikant im Vergleich zu Acker

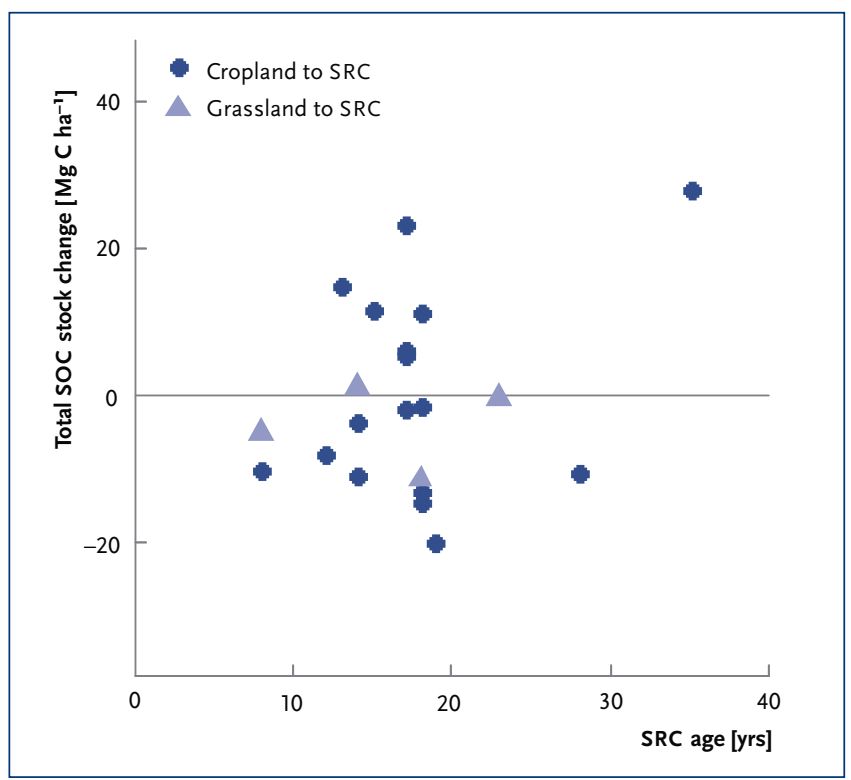

Abbildung 2: Veränderung der Vorräte an organischem Bodenkohlenstoff in 0-80 cm in Abhängigkeit des KUP-Alters

(Quelle: Walter, Don und Flessa 2015, Teilprojekt ÖL 3 im BEST-Abschlussbericht)

und Grünland. In dieser Hinsicht konnte auch kein Zusammenhang mit dem Alter der KUP festgestellt werden (Abbildung 2). Bei der Landnutzungsänderung von Acker zu KUP variiert die Änderung standortabhängig von einer jährlichen Abnahme von $1,3 \mathrm{tC} \mathrm{ha}^{-1}$ bis zu einer jährlichen Akkumulation von 1,4t C ha ${ }^{-1}$. Bei Etablierung von KUP auf Grünland liegt die Spanne bei $-0,6$ bis $+0,1 \mathrm{tCha}^{-1} \mathrm{a}^{-1}$. Es kommt also eher $\mathrm{zu}$ Verlusten von OBK bei Umwandlung von Grünland in KUP als zur OBK-Akkumulation. Obwohl der OBK-Vorrat selbst vom Tongehalt abhängt, konnte kein Zusammenhang zwischen der Veränderung des OBK-Vorrats und dem Tongehalt - oder anderen abiotischen Faktoren festgestellt werden.

\subsection{Landschaftspflegeholz}

Im Zusammenhang mit der Mobilisierung zusätzlicher Holzreserven sind Straßenbegleithölzer und Landschaftspflegeholz von großem Interesse. Dazu wurden sämtliche Offenlandgehölze, insgesamt 61.029 Objekte, des Landkreises Göttingen digitalisiert. Die Erhebungen für den Landkreis Göttingen ergaben, dass 62,7\% aller Gehölze beziehungsweise 77,5\% der vorhandenen Biomassezuwächse über eine Straße in weniger als $5 \mathrm{~m}$ Entfernung erreichbar wären. Bei rund $63 \%$ dieser Gehölze wäre jedoch die Anfahrt auf unbefestigten Straßen unumgänglich. Während rund $40 \%$ der erreichbaren Gehölze Einzelobjekten zugehören, handelt es sich nur bei rund $5 \%$ um flächige Gehölze und bei rund $18 \%$ um lineare Strukturen in der Landschaft. Diese tragen jedoch mit rund $47 \%$ erheblich zur potenziellen Biomasse aus den gesamten erreichbaren $\mathrm{Ge}$ hölzen bei, sodass ihre systematische Nutzung eine realistische Option wäre. Einzelne Objekte wie Bäume oder Büsche sind hingegen von geringer Bedeutung (3\%). Den höchsten ener- 
getischen Nutzen hätten zuwachsstarke und flächenhafte Gehölze, die zwar selten sind, jedoch insgesamt rund $50 \%$ der $\mathrm{Zu}$ wächse der erreichbaren Gehölze erbringen.

\subsection{Schwachholzbiomasse}

Der Projektteil "Schwachholzbiomasse“ verfolgte das Ziel, die Möglichkeiten und Grenzen einer nachhaltigen Nutzung von bisher ungenutztem Waldrestholz in den Modellregionen "Landkreis Göttingen“ und „Thüringer Ackerebene“ aufzuzeigen. In den Potenzialabschätzungen wurden sowohl unterschiedliche Bewirtschaftungsintensitäten und Holzsortierungen als auch die mit einer Intensivierung der Holznutzung unter Umständen verbundenen Nährstoffentzüge berücksichtigt, um eine Nährstoffnachhaltigkeit sicherzustellen. Zudem wurde in diesem Teilprojekt eine praxisnahe Methode zur Erfassung der Holzqualitäten im Bestand entwickelt, die als Grundlage für eine Optimierung der Sortierung und daher auch zu Potenzialanalysen diente. Die Ergebnisse bestätigten auch für bislang wenig untersuchte Baumarten, dass sich die meisten Nährstoffe in der Rinde und im Kronenmaterial befinden. Mit zunehmendem Rindenanteil beziehungsweise mit abnehmendem Astdurchmesser nimmt der Nährstoffentzug durch die Holzernte daher überproportional zu. Die aus energetischer Sicht angestrebte Nutzung der schwächeren Holzsortimente sollte also nur dort stattfinden, wo auf Dauer eine Verschlechterung des Nährstoffzustands der Standorte auszuschließen ist.

\subsection{Anbausysteme}

Ein weiterer thematischer Cluster von BEST widmete sich innovativen Anbausystemen zur Erzeugung von holziger Energie-Biomasse. Ein Ziel bestand beispielsweise darin aufzuzeigen, welche stofflich-ökologischen Folgewirkungen durch die Integration holziger Pflanzen zur Erzeugung von Energiebiomasse in landwirtschaftliche Produktionslinien entstehen können. Interessante Ergebnisse wurden besonders hinsichtlich des Stickstoffhaushalts neuartiger Biomasseproduktionssysteme erzielt. Es zeigte sich, dass KUP-Systeme mit Weide den Stickstoffumsatz im frühen Stadium aktivieren. Dies kann ein erwünschter Effekt auf stickstoffarmen oder degradierten Böden sein, auf denen KUPs begründet werden sollen. Mit diesem an sich vorteilhaften Effekt kann jedoch eine erhöhte Nitratauswaschung einhergehen. Durch Anbau von KUPs im Wechsel mit Grünlandstreifen („Alley Cropping“) kann dieser Effekt jedoch abgemildert werden. Weiden-KUPs erwiesen sich wegen der hohen Stickstoffeffizienz als geeignetstes System für ärmere Standorte. Pappeln zeigten hingegen die stärksten Aufnahmeraten von $\mathrm{N}$ und die höchste Biomasseproduktivität unter günstigen Nährstoffbedingungen. KUPs beider Baumarten können den Untersuchungen zufolge effizient dazu beitragen, unerwünschte Stickstoffausträge im Sickerwasser zu verhindern oder zu mindern.

Im Teilprojekt „Grünlandbiomasse“ sollten im Rahmen eines neu etablierten Agroforstsystems aus Weiden im Kurzum- trieb und aus Grünland unterschiedliche Grünlandvarianten hinsichtlich ihrer Bestandsdynamik, ihres Biomasseertrags und ihres Energieertrags bei verschiedenen energetischen Konversionsverfahren untersucht werden. Die Ergebnisse zeigten deutlich, dass während der Etablierungsphase der ersten Rotation der Weiden der Biomasseertrag durch die Grünlandanteile im Agroforstsystem gesteigert werden konnte. Mit diesem Befund wurde bei der Suche nach optimierten KUP-Varianten eine wichtige Zielsetzung erreicht: Fehlende Erträge von KUPFlächen während der Etablierung beziehungsweise der ersten Rotation können, zumindest teilweise, durch Grünlandanteile im Rahmen eines Agroforstsystems ausgeglichen werden.

Aufgabe des Teilprojekts „Bewässerung“ war die Untersuchung der Chancen und Risiken der Nutzung von biologisch vorgeklärtem Abwasser für die Holzproduktion in KUPs. Die BEST-Beispielregion Thüringer Ackerebene (BERTA) gehört zu den trockensten Gebieten Deutschlands. Im Zuge des prognostizierten Klimawandels soll sich die Trockenheit im Sommerhalbjahr in dieser Region noch verstärken. Zur Milderung des vorhandenen Wasserdefizits ist der Einsatz von biologisch geklärtem Abwasser für die Bewässerung von KUP daher eine interessante Option. Dadurch wäre es möglich, Wasser, das sonst über die Vorfluter in die Flüsse ablaufen würde, in der Region zu halten und produktiv im Sinne der Biomasseerzeugung einzusetzen. Die bewässerten Bestände auf den Thüringer Flächen bildeten deutlich mehr Biomasse als die unbewässerten, wobei auch hier Baumartenunterschiede deutlich wurden. In Tabelle 2 ist der Vorratsunterschied zwischen den bewässerten und unbewässerten Beständen dargestellt. Auf BERTA II ist der Vorratsunterschied zwischen den bewässerten und unbewässerten Baumarten im gesamten Versuchszeitraum sehr stark angestiegen.

\subsection{Bio-Kraftstoff}

Hier sollten Holzaufschlussverfahren und die Verwertbarkeit verschiedener Baumarten zur enzymatischen Hydrolyse der Holzpolysaccharide für eine biotechnologische Produktion von Bioethanol erprobt werden. Ein besonderer Schwerpunkt der Untersuchungen lag dabei auf Holz von schnell wachsenden Baumarten sowie auf Rest- und Abfallholz aus der holzverarbeitenden Industrie. Das notwendige Holzaufschlussverfahren und die folgende biotechnologische Holzhydrolyse sollten in Abhängigkeit vom Ausgangszustand des Rohmaterials (u.a. Baumart, Rindenanteile und Vornutzung) angepasst und opti-

\begin{tabular}{lccc}
\hline & $\mathbf{2 0 1 1}[\%]$ & $\mathbf{2 0 1 2}[\%]$ & $\mathbf{2 0 1 3}[\%]$ \\
\hline Pappel, BERTA I & 7,0 & 9,2 & 7,7 \\
\hline Weide BERTA I & 33,5 & 33,7 & 34,2 \\
\hline Pappel BERTA II & 51,2 & 158,3 & 168,4 \\
\hline Weide BERTA II & 104,1 & 313,8 & 664,1 \\
\hline
\end{tabular}

Tabelle 2: Vorratsdifferenz zwischen bewässerten und unbewässerten Versuchsflächen (Quelle: Lorenz und Müller 2015, Teilprojekt IO-A 4 im BEST-Abschlussbericht) 
miert werden. Nach enzymatischer Hydrolyse (EH) aller Proben zur Umsetzung von Cellulose zu Glucose fiel auf, dass sich zwischen den Laubhölzern Pappel, Weide und Buche keine signifikanten Unterschiede in der Glucose-Ausbeute zeigen. Die Dauer der EH sollte mindestens 60 min betragen. Weizenstroh zeigte dagegen schon nach einer Vorbehandlungszeit von 30 Minuten eine hohe Umsetzung von über $80 \%$ der Cellulose, im Vergleich zu ca. 40-50\% bei den Laubhölzern. Nadelholz (Fichte) scheint für den entwickelten Prozess gänzlich ungeeignet zu sein, da auch nach einer Vorbehandlungsdauer von 120 Minuten nur eine minimale Umsetzung von ca. 10\% Cellulose erreicht wird.

\subsection{Holzwerkstoffe und Kaskadennutzung}

Eines der Cluster in BEST beschäftigte sich speziell mit Holzwerkstoffen und verglich konventionell hergestellte Spanund Faserplatten sowie Holz-Kunststoff-Komposite mit solchen Produkten, die unter Verwendung von Hölzern aus KUP sowie recycelten Holzwerkstoffen hergestellt wurden. Alle getesteten Platten erfüllten die geforderten Werte gemäß geltender technischer Norm und wären damit kommerziell zu vermarkten. Ein eindeutiger Zusammenhang zwischen Rohstofftyp und Platteneigenschaften war nicht erkennbar. Auch die alternativ erzeugten Komposite zeigten gute und für die praktische Verwendung geeignete technologische Eigenschaften. Die stoffliche Verwendung beziehungsweise Wiederverwendung von Hölzern vor ihrer endgültigen energetischen Nutzung eröffnet damit auch für Hölzer von ursprünglich minderem Wert die interessante Perspektive der „Kaskadennutzung“. Dabei kann eine insgesamt wesentlich höhere Wertschöpfung als bei unmittelbarer energetischer Nutzung der holzigen Biomasse realisiert werden.

\section{8 Ökologische Folgenabschätzung}

In diesem thematischen Cluster waren diejenigen Teilprojekte zusammengefasst, die sich in BEST mit den Auswirkungen der Anbauverfahren auf die Lebensgemeinschaften und Ökosysteme beschäftigten. Die Erhaltung der Biodiversität in der modernen, multifunktionalen Agrarlandschaft ist eines der Kernziele einer nachhaltigen Bewirtschaftungsweise. Die Untersuchungen zu den ökologischen Auswirkungen der Bioenergie-Erzeugung auf die Biodiversität waren daher eine wichtige Voraussetzung für eine umfassende Bewertung von Nutzungssystemen.

Ein Ziel der Forschung für eine integrierte Folgenabschätzung der mit Bioenergie-Anbausystemen verbundenen Auswirkungen war zum Beispiel die Erfassung und Bewertung der Diversität der Begleitflora. In beiden BEST-Untersuchungsregionen (Landkreis Göttingen, Thüringer Ackerebene) erfolgte ein Monitoring der Begleitflora und der Habitatbedingungen (Bodenparameter, Lichtklima, Bewirtschaftungsintensität, Makroklima) von verschiedenen Bioenergie-Anbausystemen (Mais, Raps, KUP). Dieses schloss konventionell bewirtschaftete Flächen ebenso ein wie Flächen des ökologischen Landbaus oder

\section{„Für eine naturschutzfachliche Bewertung muss der gesamte Lebenszyklus einer Kurzumtriebs- plantage untersucht werden."}

Flächen, die im Rahmen von Agrarumweltmaßnahmen extensiver bewirtschaftet wurden. Die Daten zur Phytodiversität wurden im Hinblick auf die naturschutzfachliche Bedeutung der erfassten Arten (Rote-Liste-Arten, Archäophyten, Neophyten) analysiert. Die Ergebnisse zeigten, dass es notwendig ist, den gesamten Lebenszyklus einer KUP in eine naturschutzfachliche Bewertung einfließen zu lassen, da sich die Habitatbedingungen in KUP im Zeitverlauf deutlich ändern. Die Artenzusammensetzung und -vielfalt war, bei geringem Herbizideinsatz, in den ersten zwei Jahren nach Neuanlage mit jener einer Ackerbrache vergleichbar (bis zu 40 Arten je $75 \mathrm{~m}^{2}$ ). Auf den alten KUP, auf denen bereits der Kronenschluss erreicht war, konnten dagegen nur geringe Artenzahlen nachgewiesen werden (bis zu 19 Arten je $75 \mathrm{~m}^{2}$ ). Arten der Roten Listen auf Landes- und auf Bundesebene wurden nicht gefunden.

\subsection{Integratives Werkzeug zur Szenarioanalyse}

Ein Produkt der Integration von Ergebnissen des Verbundprojekts stellte das Beratungswerkzeug „BEAST“ (Bioenergy Assessment and Scenario Tool) als Desktop-Anwendung zur regionalen Szenario-Potenzialanalyse für holzige Biomasse dar. Über mehrere Prototypen und nach wiederholter Einbindung potenzieller Anwender aus dem Bereich der Regionalplanung wurde die Endversion dieser Software datenbankfrei in Java implementiert. Mithilfe des Beratungswerkzeugs lassen sich über den „Szenariogenerator" Szenarien zum zukünftigen Energiebedarf sowie zu den ökonomischen und ökologischen Rahmenbedingungen für die Produktion von holziger Biomasse aus Waldholz, Landschaftspflegeholz und KUP definieren. Diese Szenarien werden anschließend in der Simulation genutzt, um daraus Biomassen- oder Energiepotenziale zu berechnen. Darüber hinaus werden diese Potenziale ökonomisch und im Fall von KUP auch ökologisch bewertet. Potenzialanalysen für die Bereiche Wald- und Landschaftspflegeholz finden nicht räumlich differenziert statt. Für den Bereich der KUP erfolgt hingegen eine räumlich explizite Allokation. Hier können von Nutzenden Flächenrestriktionen angegeben sowie Kriterien skaliert und gegeneinander gewichtet werden, die für die Auswahl und Priorisierung von potenziellen Anbauflächen für KUP Verwendung finden. Bei der ökonomischen Bewertung wird KUP mit den annuellen Ackerkulturen Raps, Winter- 


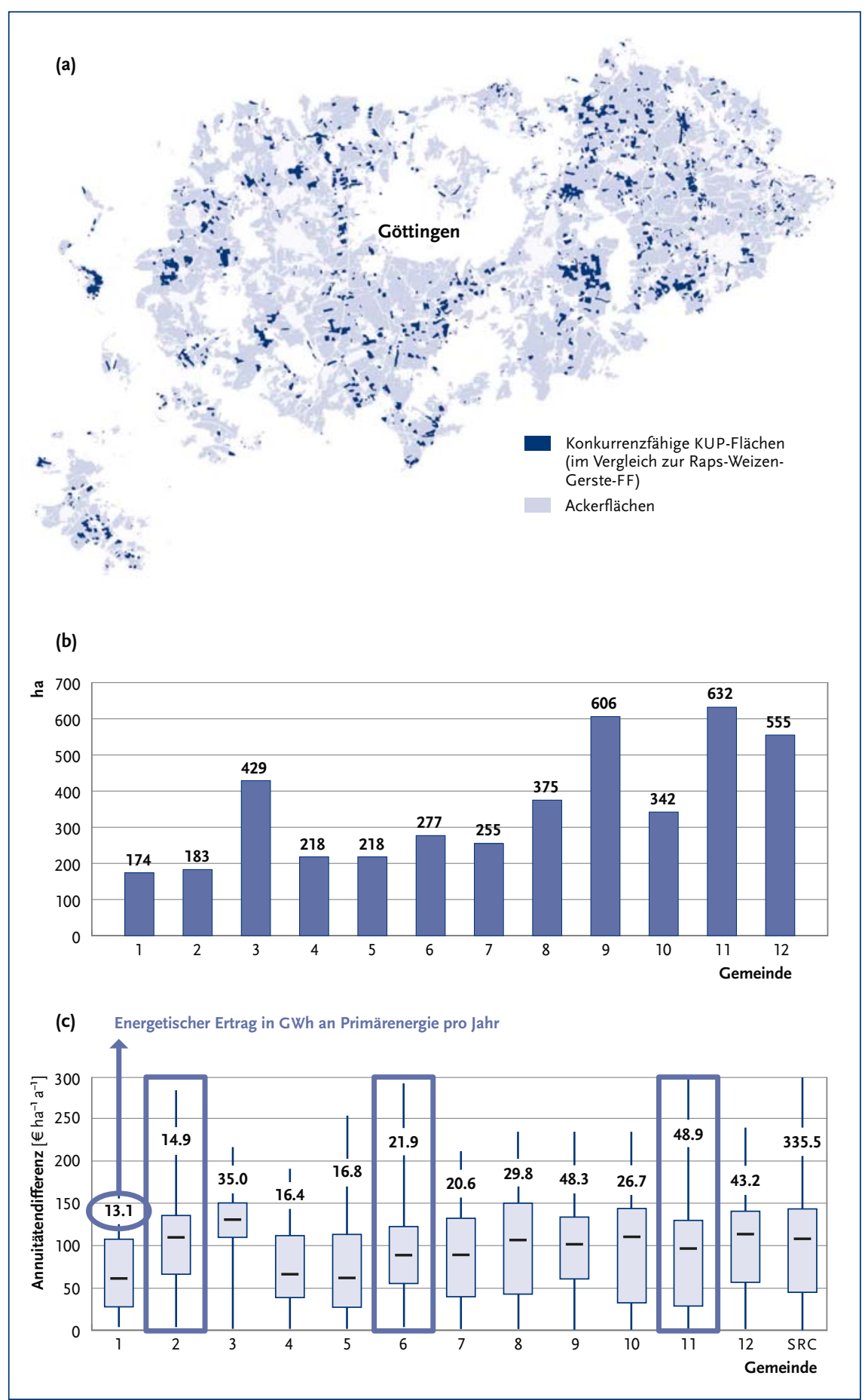

Abbildung 3:

(a) Verteilung der ökonomisch konkurrenzfähigen KUP-Flächen unter den gegebenen Szenarienbedingungen,

(b) Flächengröße an konkurrenzfähigen KUPs pro Gemeinde,

(c) Annuitätendifferenz und Primärenergiebereitstellung der KUP-Flächen pro Gemeinde

(Quelle: G. Busch 2015, Teilprojekt UP4 im BEST-Abschlussbericht) gerste und Winterweizen als Hauptanbaufrüchten des Landkreises Göttingen verglichen. Die ökologische Bewertung berücksichtigt die Wirkung von KUP im Hinblick auf den Erosionsschutz, den Grundwasserschutz und die Landschaftsstruktur.

Mit dem vorliegenden Werkzeug existiert nun eine Kombination aus ökologischer Analyse, Ertragsmodellierung und ökonomischer Bewertung in anwendungsrelevanter Komplexität, die es erlaubt, das Werkzeug in regionalen Planungs- und Diskussionsworkshops zum Themenbereich holzige Biomasse, Klimaschutz und nachhaltige Landnutzung einzusetzen. Das Beratungswerkzeug hat auf Seiten der Politik, vorgestellt in Fachausschüssen des Kreistags Göttingen, großes Interesse hervorgerufen. BEST wird unter anderem Bestandteil des neuen LEADER-Programms für regionale Auswertungen. Abbildung 3 zeigt ein Anwendungsbeispiel: Dargestellt ist ein (hypothetisches) Szenario der Etablierung von KUP im Landkreis Göttingen auf ökonomisch konkurrenzfähigen (Annuität von KUP > als RapsWinterweizen-Wintergerste-Fruchtfolge) Standorten.

Im Szenario erbringt die Gesamtfläche von 4.263 ha KUP einen jährlichen Primärenergiebeitrag von 336 GWh, das entspricht $17 \%$ des nachgefragten erneuerbaren Energieanteils in dem „ModeratSzenario“ des integrierten Klimaschutzkonzepts für den Landkreis Göttingen. Damit könnten KUPs eine interessante und relevante Erweiterung des Mixes an regional verfügbaren erneuerbaren Energieträgern darstellen.

\section{Zusammenfassung und Schlussfolgerungen}

Das Verbundprojekt BEST adressierte auf der Grundlage fachwissenschaftlicher Forschungsarbeiten und unter strenger Beachtung einer interund transdisziplinären Ausrichtung des Gesamtprojekts eine Vielzahl aktueller Problemfelder im Bereich der Energiegewinnung aus Biomasse. Konkret ging es um die Produktion und Bereitstel- 
lung holzbasierter Biomasse zur Energiegewinnung, bei der die gesamte Bandbreite der hierfür relevanten Aspekte berücksichtigt wurde. Diese reicht von der Flächenverfügbarkeit und Flächenkonkurrenzen über die ökonomische Konkurrenzfähigkeit gegenüber anderen Landnutzungsformen bis hin zur ökologischen Verträglichkeit neuer Anbauformen für Energieholz. Neben der Erfassung und Bewertung von Wald- und Landschaftspflegeholzpotenzialen standen Kurzumtriebsplantagen (KUP) mit schnell wachsenden Baumarten und deren Kombination mit einer agrarischen Nutzung, sogenannte Agroforstsysteme, im Fokus. Neben der Klärung grundlegender wissenschaftlicher Fragestellungen zur Optimierung der Biomassenutzung und zu deren ökologischen und sozio-ökonomischen Wirkungen verfolgte BEST einen stark partizipativen Ansatz. Ziel war es, die Akteur/innen und Anwender/innen von Forschungsergebnissen von Anfang an intensiv in die Projektarbeiten einzubeziehen und im Hinblick auf die einzelnen Forschungsfragen zu beteiligen. Aus diesem Dialog heraus entstanden neben den wissenschaftlichen Fachpublikationen zahlreiche Veröffentlichungen, die auf die jeweiligen Nutzer/innen zugeschnitten waren, sowie ein computergestütztes und webbasiertes Beratungswerkzeug, das eine flächenscharfe und nach unterschiedlichen Kriterien optimierbare Planung von KUP erlaubt. Darüber hinaus wurden die wichtigsten Projektergebnisse in einer internationalen Buchveröffentlichung dokumentiert.

In der Gesamtschau und Integration der Ergebnisse aus $B E S T$ lässt sich folgern, dass Biomasse aus Holz für die Energiegewinnung geeignet ist, einen bedeutenden Beitrag zu einer künftigen nachhaltigen Versorgung mit erneuerbaren Energien zu leisten. Dabei kann der Anbau von KUP, wenn er umsichtig geplant ist und Rücksicht auf standörtliche, klimatische sowie ökonomische Rahmenbedingungen nimmt, positive ökologische und ökonomische Effekte erzielen. Beispiele für vorteilhafte Wirkungen sind die Erhöhung der Habitatvielfalt, der Erosionsschutz und die Minderung von Nitratausträgen beziehungsweise Treibhausgasemissionen. Darüber hinaus konnte gezeigt werden, dass klassische Anbauformen zur Produktion von holziger Biomasse für die Energiegewinnung weiterentwickelt und optimiert werden können. Zugleich wurde erkennbar, dass ein großes Potenzial dafür vorhanden ist, die erzeugte Biomasse vor der thermischen Nutzung einer stofflichen Verwendung zuzuführen. Letztlich konnten durch das Projekt aber auch die Grenzen der Energiegewinnung aus holziger Biomasse deutlich gemacht werden. Diese ergeben sich für den Wald vor allem mit Blick auf die Nährstoffnachhaltigkeit für die erstmals regional vollständig erfassten Offenlandgehölze aus Wirtschaftlichkeitsaspekten und im Hinblick auf die KUP vor allem aus betrieblichen Hemmnissen. Darüber hinaus wurde deutlich, dass selbst unter optimistischen Annahmen eine vollständige Versorgung mit erneuerbaren Energien in Regionen wie den von BEST untersuchten voraussetzt, dass es gelingt, den gesamten Energiebedarf und -verbrauch über Einsparungen drastisch zu reduzieren. Diese Erkenntnis

\section{„Der Anbau von Kurzumtriebs- plantagen kann, wenn er Rücksicht auf standörtliche, klimatische sowie ökonomische Rahmen- bedingungen nimmt, positive Effekte erzielen."}

zeigt, dass die Bemühungen um die Bereitstellung erneuerbarer Energie von einem gesellschaftlichen und politischen Prozess begleitet werden müssen, der neben einer gesteigerten Effizienz (Produktivität je Einheit eingesetzter Ressource), Konsistenz (Entwicklung naturverträglicherer Technologien) und Permanenz (Erhöhung der Produktdauer) vor allem eine Verbesserung der Suffizienz (geringerer Verbrauch von Ressourcen) zum Ziel hat.

\section{Anmerkung}

[1] Butler-Manning, D./Bemmann, A./Bredemeier, M./Lamersdorf, N./ Ammer, C. (2015): Bioenergy from Dendromass for the Sustainable Development of Rural Areas, First Edition. Weinheim, Wiley.

\section{Literatur}

Alle zitierten Ergebnisse im Text sowie Abbildungen und Tabellen aus: Abschlussbericht (Jahre 2010-2014) zum Verbundprojekt BEST BioenergieRegionen stärken - Neue Systemlösungen im Spannungsfeld ökologischer, ökonomischer und sozialer Anforderungen. Sektion Waldökosystemforschung im Zentrum für Biodiversität und nachhaltige Landnutzung, Georg-August-Universität Göttingen.

\section{AUTOREN + KONTAKT}

Michael Bredemeier ist Professor und wissenschaftlicher Koordinator an der Sektion Waldökosystemforschung des Zentrums für Biodiversität und nachhaltige Landnutzung der Universität Göttingen.

Center for Biodiversity and Sustainable Land Use (CBL), Universität Göttingen, Büsgenweg 2, 37077 Göttingen. Tel.: +49 551 3998-40, E-Mail: mbredem@gwdg.de Norbert Lamersdorf ist Professor für Bodenkunde und Waldernährung an der Universität Göttingen.

E-Mail: nlamers@gwdg.de

Christian Ammer ist Professor für Waldbau und Waldökologie der gemäßigten Zonen an der Universität Göttingen.

E-Mail: Christian.Ammer@forst.uni-goettingen.de
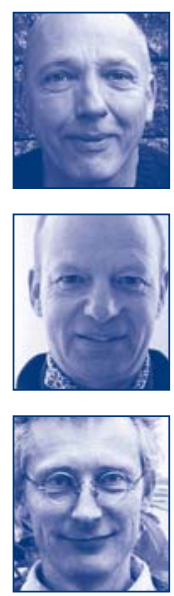